\title{
REVISÃO SISTEMÁTICA DA LITERATURA: CONCEITUAÇÃO, PRODUÇÃO E PUBLICAÇÃ̃O
}

\author{
Maria Cristiane Barbosa Galvão \\ Universidade de São Paulo (USP) \\ mgalvao@usp.br \\ Ivan Luiz Marques Ricarte \\ Universidade Estadual de Campinas (UNICAMP) \\ ricarte@unicamp.br
}

\begin{abstract}
Resumo
Realizar uma revisão sistemática de literatura vai além da atividade usual de fazer uma revisão de literatura como parte de um trabalho de pesquisa acadêmica. A revisão sistemática é uma modalidade de pesquisa, que segue protocolos específicos e busca dar alguma logicidade a um grande corpus documental. Este artigo apresenta os aspectos essenciais no desenvolvimento de revisões sistemáticas da literatura. Após a conceituação e apresentação dos diferentes tipos de revisão sistemática, são abordadas as etapas de sua produção, desde a delimitação da questão de pesquisa, a seleção das bases de dados, a elaboração da estratégia de busca, a seleção dos documentos e a sistematização dos resultados. Por fim, são abordados aspectos relacionados à divulgação e publicação de revisões sistemáticas de literatura. A compreensão desses aspectos é essencial não apenas para autores desse tipo de pesquisa, mas também para revisores e editores de periódicos de todas as áreas do conhecimento.
\end{abstract}

Palavras-chave: Revisão sistemática. Metodologia.

\section{SYSTEMATIC LITERATURE REVIEW: CONCEPT, PRODUCTION AND PUBLICATION}

\begin{abstract}
Performing a systematic review goes beyond the usual activity of performing a literature review as part of an academic research. Systematic review is a type of research, which follows specific protocols and aims to give some logic to a large documentary corpus. This article presents the essential aspects in the development of systematic reviews of literature. After the conceptualization and presentation of the different types of systematic reviews, the stages of its production are approached, from the delimitation of the research question, the selection of databases, the elaboration of search strategies, the selection of documents and the systematization of results. Finally, aspects related to the dissemination and publication of systematic reviews are addressed. Understanding these aspects is essential not only for authors of this type of research, but also for journal reviewers and editors of all fields of knowledge.
\end{abstract}

Keywords: Systematic review. Methodology.

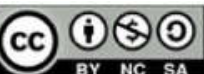

Esta obra está licenciada sob uma Licença Creative Commons Atribuição 4.0 Internacional (CC BY-NC-AS 4.0). LOGEION: Filosofia da informação, Rio de Janeiro, v. 6 n. 1, p.57-73, set.2019/fev. 2020 


\section{INTRODUÇÃO}

Revisar a literatura é atividade essencial no desenvolvimento de trabalhos acadêmicos e científicos. A realização de uma revisão de literatura evita a duplicação de pesquisas ou, quando for de interesse, o reaproveitamento e a aplicação de pesquisas em diferentes escalas e contextos. Permite ainda: observar possíveis falhas nos estudos realizados; conhecer os recursos necessários para a construção de um estudo com características específicas; desenvolver estudos que cubram brechas na literatura trazendo real contribuição para um campo científico; propor temas, problemas, hipóteses e metodologias inovadoras de pesquisa; otimizar recursos disponíveis em prol da sociedade, do campo científico, das instituições e dos governos que subsidiam a ciência. Não por acaso, artigos que apresentam revisões de literatura estão usualmente entre os mais procurados pelos leitores de publicações científicas (BAEK et al., 2018).

Revisão de literatura é um termo genérico, que compreende todos os trabalhos publicados que oferecem um exame da literatura abrangendo assuntos específicos. É possível encontrar diversos artigos de revisão de literatura que apresentam diferentes abordagens para as diferentes etapas do desenvolvimento desses trabalhos. Grant e Booth (2009) identificaram 14 diferentes tipos de revisão de literatura, variando desde a visão geral até as revisões sistemáticas e meta-análises. Porém, uma diferenciação básica precisa ser estabelecida entre a revisão de literatura de conveniência e a revisão sistemática da literatura.

A revisão de conveniência é aquela na qual o pesquisador reúne e discorre sobre um conjunto de trabalhos científicos que julga importante para o tratamento de uma temática, mas não apresenta critérios explícitos sobre como a revisão foi construída para que possa ser reproduzida por outros pesquisadores. Esta modalidade de revisão pode ser empregada em diferentes situações: na introdução de um trabalho de conclusão de curso, de uma dissertação de mestrado ou de uma tese de doutorado, no editorial de um periódico, em um artigo de opinião, em um artigo com fins educacionais ou de divulgação científica. Porém, considerando a falta de explicitação de critérios em sua elaboração, essa modalidade de revisão de literatura possui baixo nível de evidência científica.

A revisão sistemática da literatura vai muito além disso. É uma modalidade de pesquisa, que segue protocolos específicos, e que busca entender e dar alguma logicidade a um grande corpus documental, especialmente, verificando o que funciona e o que não funciona num dado contexto. Está focada no seu caráter de reprodutibilidade por outros pesquisadores, apresentando de forma explícita as bases de dados bibliográficos que foram consultadas, as 
estratégias de busca empregadas em cada base, o processo de seleção dos artigos científicos, os critérios de inclusão e exclusão dos artigos e o processo de análise de cada artigo. Explicita ainda as limitações de cada artigo analisado, bem como as limitações da própria revisão. De forma geral, a revisão de literatura sistemática possui alto nível de evidência e se constitui em um importante documento para tomada de decisão nos contextos públicos e privados. Dito de outro modo, a revisão sistemática de literatura é uma pesquisa científica composta por seus próprios objetivos, problemas de pesquisa, metodologia, resultados e conclusão, não se constituindo apenas como mera introdução de uma pesquisa maior, como pode ser o caso de uma revisão de literatura de conveniência.

Pelo exposto, este artigo tem três objetivos: apresentar a conceituação relacionada ao termo revisão sistemática da literatura; apresentar seus processos de produção e protocolos mais conhecidos; e, finalmente, discutir alguns aspectos relacionados à publicação das revisões sistemáticas.

\section{REVISÃO SISTEMÁTICA DA LITERATURA: CONCEITUAÇÃO}

Considerando que existem diferentes campos do conhecimento, diversos autores têm buscado equacionar as tipologias possíveis de revisões sistemáticas. Assim, Siddaway, Wood e Hedges (2019) classificam as revisões sistemáticas em revisões sistemáticas com metaanálise; revisões sistemáticas narrativas; e revisões sistemáticas com meta-síntese.

Segundo os autores, quando o revisor deseja reunir muitos estudos que testaram empiricamente a mesma hipótese, é necessária uma revisão quantitativa, denominada de metaanálise. Assim, a meta-análise está preocupada com: a estimativa; o relato de resultados quantitativos semelhantes; o exame dos mesmos construtos e relacionamentos; ou seja, analisa os estudos que seguem o mesmo desenho de pesquisa quantitativa. (SIDDAWAY; WOOD; HEDGES, 2019)

Já uma revisão narrativa, segundo os autores, é apropriada quando os estudos quantitativos a serem considerados empregam diversas metodologias ou partem de diferentes conceituações teóricas, construtos e/ou relacionamentos. As revisões narrativas sintetizam os resultados de estudos quantitativos individuais sem referência à significância estatística dos resultados. Elas são um meio particularmente útil de unir estudos sobre diferentes tópicos para reinterpretação ou interconexão, a fim de desenvolver ou avaliar uma nova teoria. As revisões narrativas também podem ser usadas para fornecer uma descrição histórica do desenvolvimento da teoria e da pesquisa sobre um tópico (SIDDAWAY; WOOD; HEDGES, 2019). 
Finalmente, a meta-síntese, também denominada de meta-etnografia e/ou meta-análise qualitativa é apropriada quando uma revisão visa integrar a pesquisa qualitativa. O objetivo de uma meta-síntese é sintetizar estudos qualitativos sobre um tópico a fim de localizar temas, conceitos ou teorias-chave que forneçam novas ou mais poderosas explicações para o fenômeno sob análise (SIDDAWAY; WOOD; HEDGES, 2019).

Além da classificação citada, muitos pesquisadores têm buscado mais intensamente a construção de revisão de literatura sistemática de caráter misto, ou seja, aquela que identifica, seleciona, avalia e sintetiza simultaneamente estudos qualitativos, estudos quantitativos e estudos mistos. Esta abordagem deriva do fato de que muitas vezes os dados quantitativos carecem de complementos para sua compreensão que podem ser encontrados em relatos presentes em estudos qualitativos, por exemplo. Já os dados qualitativos nem sempre suportam a generalização de resultados para uma grande população, necessitando, muitas vezes, do suporte dos dados quantitativos. Assim, os adeptos dos métodos mistos de pesquisa e das revisões mistas de literatura vislumbram que a complexidade do século 21 exige uma visão mais cooperativa e integrada das diferentes ciências e seus métodos. São expoentes nessa abordagem Creswell e Clark (2010), assim como Pope, Mays e Popay (2007).

Galvão, Pluye e Ricarte (2017) sintetizam quatro tipos de revisões mistas, resumidos a seguir.

A revisão mista de convergência quantitativa é aquela que transforma os resultados dos estudos qualitativos, quantitativos e de estudos empregando métodos mistos em achados quantitativos (sejam variáveis ou valores). Esse tipo de revisão é aplicável quando os estudos selecionados mencionam grande número de participantes.

A revisão mista de convergência qualitativa é aquela que transforma os resultados dos estudos qualitativos, estudos quantitativos e de estudos empregando métodos mistos em achados qualitativos (por exemplo, em temas). Esse tipo de revisão é aplicável quando os estudos analisados possuem amostras pequenas e estão voltados para desenvolver, refinar e revisar um quadro conceitual, por exemplo.

A revisão mista sequencial exploratória é composta por duas etapas. $\mathrm{Na}$ etapa 1 , os resultados dos estudos qualitativos, quantitativos e dos estudos empregando métodos mistos são transformados em achados qualitativos usando, por exemplo, a análise temática. Na etapa 2 , os resultados quantitativos são tabulados e comparados, desde que haja uma entidade comum entre os estudos quantitativos.

A revisão mista sequencial explanatória é empregada nos casos onde se quer medir os efeitos de ações, intervenções ou programas (etapa 1) e explicar diferenças em seus efeitos 
(etapa 2). Nessa modalidade de revisão, a integração ocorre entre as etapas quantitativa e qualitativa, na medida em que a síntese quantitativa (etapa 1) fornece subsídios para a síntese qualitativa (etapa 2), e na interpretação dos achados das duas etapas.

Em que pese a explanação apresentada sobre os diferentes tipos de revisão sistemática de literatura, é fato que a qualidade das revisões publicadas ao redor do mundo é muito variável, podendo gerar muita confusão para estudantes e pesquisadores iniciantes. Nesse sentido, ferramentas têm sido desenvolvidas para auxiliar na verificação dos critérios mínimos de qualidade das revisões de literatura, antes, durante e após publicação.

Uma delas é o Preferred Reporting Items for Systematic Reviews and Meta-Analyses, bastante conhecida como PRISMA (2015), que traz uma lista dos itens que devem estar presentes em uma revisão sistemática (PRISMA checklist), assim como apresenta o fluxo dos critérios de inclusão e exclusão de artigos de uma revisão sistemática (PRISMA flow diagram). No checklist do PRISMA, por exemplo, podem ser encontrados itens como: o título indica de que se trata de uma revisão sistemática ou uma meta-análise ou ambas?; traz nos objetivos uma questão clara, ou seja, contendo as pessoas ou o problema que será abordado na revisão, o tipo de intervenção que será analisado, se haverá comparação entre diferentes intervenções e quais serão os resultados (outcomes) analisados nos estudos selecionados? há indicação de que a revisão sistemática segue um protocolo e foi registrada em uma plataforma de revisões?; indica quais foram os critérios para a inclusão dos estudos na revisão?; indica quais foram as fontes de informação empregadas, incluindo pelo menos uma base de dados bibliográfica, e explicita como foram realizadas as estratégias de busca empregadas?; explicita como foi o processo de leitura e a aplicação dos critérios de seleção foram realizados?; apresenta o número de documentos selecionados em cada fase em forma de fluxo?; indica as limitações da revisão e potenciais vieses?; apresenta um resumo com foco no grande público e tomadores de decisão?; apresenta informação sobre financiamento ou apoio para o desenvolvimento da revisão?

Outra ferramenta usualmente utilizada para verificar a adequação de uma revisão sistemática é o CASP Systematic Review Checklist (CRITICAL, 2018), que propõe uma lista de questões para verificar uma revisão sistemática, refletindo sobre os resultados apresentados, a validade desses resultados e sua aplicabilidade local. As questões são: a revisão abordou uma questão claramente focada?; os autores procuraram os tipos certos de artigos?; você acha que todos os estudos importantes e relevantes foram incluídos?; os autores da revisão fizeram o suficiente para avaliar a qualidade dos estudos incluídos?; se os resultados da revisão foram combinados, foi razoável fazê-lo?; quais são os resultados gerais da revisão?; qual é a precisão dos resultados?; os resultados podem ser aplicados à população local?; todos os resultados 
importantes foram considerados?; os benefícios apresentados superam os danos e os custos envolvidos? Para cada questão, há sugestões para auxiliar a refletir sobre a resposta que será dada. Por exemplo, na primeira questão, sugere-se que o foco seja avaliado em termos da população estudada, a intervenção aplicada e o resultado considerado, ou seja, se tem os elementos essenciais de uma questão.

No contexto das revisões mistas, uma ferramenta para analisar a qualidade é a Mixed Methods Appraisal Tool (MMAT), desenvolvida por Hong et al. (2018), que lista os elementos que devem estar presentes em estudos e revisões mistas. No MMAT podem ser observados itens para avaliação como: há perguntas de pesquisa claras?; os dados coletados permitem abordar as questões da pesquisa?; existe uma justificativa adequada para se desenvolver uma revisão de literatura empregando métodos mistos?; os diferentes componentes do estudo estão efetivamente integrados para responder à pergunta da pesquisa?; os resultados da integração de componentes qualitativos e quantitativos são adequadamente interpretados?; as divergências e inconsistências entre os resultados quantitativos e qualitativos são adequadamente tratadas?; os diferentes componentes do estudo aderem aos critérios de qualidade de cada tradição dos métodos envolvidos?

Referente ainda à qualidade das revisões, a Classificação dos Níveis de Evidência de Oxford, construída por Howick et al. (2012), entende que as revisões sistemáticas de estudos transversais, as revisões sistemáticas de estudos de coorte e as revisões sistemáticas de ensaios clínicos randomizados possuem nível 1 de evidência, em uma escala de 1 a 5 , onde 1 é o nível de maior evidência. Assim, a classificação de Oxford ressalta a importância das revisões sistemáticas para o avanço científico e para as diferentes tomadas de decisão, sobretudo no contexto da saúde.

\section{REVISÃO SISTEMÁTICA DE LITERATURA: PRODUÇÃO}

Conforme apresentado no tópico anterior, as revisões sistemáticas seguem protocolos específicos. Assim, a fim de contribuir para que as revisões de literatura ganhem em qualidade, serão discutidas, neste tópico, algumas etapas que compõem seu desenvolvimento, como a delimitação da questão a ser tratada na revisão; a seleção das bases de dados bibliográficos para consulta e coleta de material; a elaboração de estratégias para busca avançada; a seleção de textos e sistematização de informações encontradas; bem como serão apresentados alguns aspectos sobre a composição da equipe para elaboração da revisão. 


\subsection{A DELIMITAÇÃO DA QUESTÃO}

Do mesmo modo que as demais pesquisas científicas, a revisão de literatura demanda a delimitação de objetivos e questões de pesquisa. Geralmente, entende-se que a questão de uma revisão sistemática deve contemplar a especificação da população, ou do problema ou da condição que será estudada, o tipo de intervenção que será analisado, se haverá comparação entre intervenções e o desfecho que se pretende estudar. Esta abordagem para a elaboração da questão é conhecida pela sigla PICO, onde p é população ou problema, i é intervenção, c é comparação e o é outcome/resultado.

Assim, tem-se: "Qual a efetividade da contação de histórias para despertar o interesse pela leitura em crianças de idade escolar?”, onde criança em idade escolar é a população, contação de histórias é a intervenção, e o interesse pela leitura é o desfecho a ser analisado na revisão de literatura. Um segundo exemplo: "Disciplinas sobre metodologia científica melhoram o desempenho de alunos de pós-graduação na elaboração de projetos de pesquisa?", onde alunos de pós-graduação é a população, disciplinas sobre metodologia científica é a intervenção, e desempenho na elaboração de projetos de pesquisa é o desfecho.

Algumas revisões de literatura partem de questões focadas na comparação entre diferentes intervenções. Assim, tem-se o exemplo: "Qual o melhor equipamento para alunos de graduação buscarem informações acadêmicas: computadores ou dispositivos móveis?’. Neste exemplo, alunos de graduação formam a população; dispositivo móvel é uma intervenção, computador é outra intervenção; e busca por informação acadêmica é o desfecho a ser estudado.

No caso das revisões de literatura mistas, geralmente, parte-se de duas questões: uma questão quantitativa e uma questão qualitativa. Assim, tem-se: "Questão quantitativa: qual a porcentagem de alunos de graduação que usam computadores ou dispositivos móveis na busca por informações acadêmicas nas diferentes regiões do Brasil? Questão qualitativa: qual a percepção de alunos de graduação sobre o uso de computadores e o uso de dispositivos móveis na busca por informações acadêmicas nas diferentes regiões do Brasil”.

O processo para se chegar à questão que norteará a revisão exige do pesquisador uma análise prévia da literatura existente. Recomenda-se para a economia de tempo e recursos que o pesquisador verifique, por exemplo, se uma revisão ou várias revisões sobre o assunto já não foram realizadas por outros pesquisadores. Caso já exista uma revisão sistemática, é possível, por exemplo, optar por sua atualização, ao invés de construir uma revisão inteiramente nova. De qualquer forma, a existência de uma questão bem delimitada será essencial para que as demais etapas da revisão de literatura sejam elaboradas. 


\subsection{A SELEÇÃO DAS BASES DE DADOS}

Delimitada a questão que será tratada na revisão, é preciso definir quais bases de dados serão consultadas para a busca de artigos e outros materiais bibliográficos que possam ser incluídos ou excluídos da revisão de literatura que se pretende realizar. Anualmente, surgem novas e mais complexas bases de dados. No entanto, algumas bases têm se consolidado no que tange ao desenvolvimento de revisões de literatura, entre elas estão:

- SciELO. Compreende a produção de artigos produzidos em vários países da América Latina;

- LISA (Library and Information Science Abstracts). Abrange a literatura internacional na área de Ciência da Informação desde 1969;

- LISTA (Library, Information Science \& Technology Abstracts). Abrange a literatura internacional nas áreas de Ciência e de Tecnologia da Informação desde meados da década de 1960;

- SCOPUS. Compreende várias áreas do conhecimento, incluindo: análise bibliométrica, história, educação, psicologia, direito, religião, linguística e literatura;

- ERIC (Education Resources Information Center). Abrange artigos e relatórios em inglês na área de educação;

- PsycINFO. Abrange a literatura internacional sobre aspectos a ciência social e comportamental, incluindo pesquisas acadêmicas em psicologia e áreas afins;

- IEEE Xplore. Abrange a coleção de trabalhos na área de tecnologia publicados pelo Institute of Electrical and Electronic Engineers;

- ACM Digital Library. Compreende trabalhos da área de Tecnologia da Informação desde a década de 1950;

- Medline Ovid. Abrange a literatura internacional de todas as especialidades médicas de 1946 até os dias atuais;

- PubMed. Abrange a literatura internacional de todas as especialidades médicas de 1966 até os dias atuais;

- Embase. Abrange a literatura biomédica internacional de 1947 até os dias atuais, incluindo estudos sobre produtos farmacêuticos, farmacovigilância, eficácia, medicamentos e dispositivos médicos; 
- CINAHL. Abrange a literatura internacional sobre enfermagem, tecnologia e áreas afins.

Embora possa haver alguma duplicação de artigos em diferentes bases de dados, cada base se destina a um público-alvo, possui uma cobertura de tipos de documentos e uma cobertura temática, ou seja, conteúdos informacionais que são por ela tratados de forma preferencial. Portanto, deriva-se que é preciso buscar a informação relevante em bases de dados adequadas e compatíveis com a temática a ser desenvolvida.

As bases de dados de acesso restrito são acessíveis internamente em institutos de pesquisa e universidades. Igualmente, o Portal Brasileiro de Informação Científica, mais conhecido como Portal de Periódicos da Coordenação de Aperfeiçoamento de Pessoal de Nível Superior (Capes), disponibiliza bases de dados de acesso restrito, mediante convênio institucional. Nesse Portal há ainda expressiva quantidade de livros integrais, artigos de periódicos, patentes, normas, teses e dissertações, e revistas internacionais e nacionais de todas as áreas do conhecimento (COORDENAÇÃO, 2019).

Para a revisão de literatura que envolva tema muito recente, tema pouco estudado como algumas populações vulneráveis, temáticas latino-americanas e africanas, temáticas relacionadas a comunidades locais, doenças tropicais, é recomendável também que, além das bases já citadas, sejam consultados os repositórios de teses e dissertações dos diferentes países, assim como o buscador Google Acadêmico, pois costumam ser mais abrangentes. Esses recursos também podem ser úteis quando se pretende fazer uma revisão sistemática de caráter exaustivo, ou seja, incluindo artigos científicos, livros, teses, dissertações e literatura cinzenta (publicações realizadas em eventos, publicações em editores não comerciais, publicações realizadas em sites pessoais de pesquisadores).

Revisões que foquem inovação tecnológica devem considerar ainda as bases de patentes, marcas e desenho, como aquelas disponibilizadas pelo Instituto Nacional de Propriedade Intelectual (INSTITUTO, 2009).

\subsection{ELABORAÇÃO DA ESTRATÉGIA DE BUSCA}

Para o uso das bases de dados bibliográficos, é necessária a montagem de uma estratégia de busca que envolve um conjunto de procedimentos e mecanismos tecnológicos existentes para localizar a informação. Grosso modo, uma base de dados possui formulários simples e formulários avançados de buscas. Os avançados permitem a busca de informação por todos os 
campos da base de dados ou por campos específicos, como título do documento, resumo do documento, autor, assunto do documento, periódico no qual o documento foi publicado, data de publicação, país de publicação, idioma de publicação, tipo de publicação (livro, anais de eventos, artigos de periódicos, teses e dissertações, normas, imagens, filmes etc.), e disponibilidade (acesso livre, acesso restrito etc.). Além destas possibilidades, algumas bases de dados permitem a busca de documentos baseada no número de citações que tiveram, ou seja, pelo nível de relevância acadêmica que possuem; busca de documentos por semelhança, isto é, uma vez que se localizou um documento de interesse, é possível recuperar outros textos com conteúdo próximo; e busca por navegação, ou seja, diante de um registro bibliográfico, podese navegar na base de dados procurando documentos do mesmo autor, com assuntos semelhantes, que citam o trabalho registrado ou que foram publicados em um mesmo periódico. Enfim, bases de dados possuem possibilidades variadas e se aperfeiçoam com frequência. No entanto, o foco deve ser a elaboração da estratégia de busca avançada, pois tal estratégia de busca é reproduzível.

Para empregar estratégias de busca avançadas uma etapa necessária é a consulta a terminologias, tesauros e dicionários especializados para realizar o mapeamento de sinônimos, assim como para traduzir adequadamente os conceitos que integram a questão de revisão para a língua inglesa, visto que as bases de dados bibliográficos internacionais priorizam a língua inglesa como idioma de busca.

Assim, dada uma questão de revisão sobre "quais os efeitos da intervenção dietética e de exercícios físicos na perda de peso e composição corporal em mulheres obesas na pósmenopausa" seria preciso levantar potenciais sinônimos e termos em língua inglesa como: diet, dietary, exercise, obese, obesity, overweight, body weight, body mass index, fat mass, perimenopause, postmenopause, menopause. Se a revisão de literatura envolver vários idiomas é recomendável realizar o levantamento dos sinônimos em todos os idiomas que serão tratados na revisão.

Dada outra questão de revisão sobre "como engajar pessoas sem moradia na área da saúde empregando tecnologias da informação e comunicação?" seria preciso levantar potenciais sinônimos e termos em língua inglesa como: homeless, unstable housing, unstably housed, housing stability, supportive housing, computer, computers, technologies, communication technology, technology, mobile technologies, ipad, handheld, mobile computing, smart phone, mobile phone, iphone, android, app, sms, text message, mobile technology, personal digital assistant, tablet computer, informatics, Internet, online, Mhealth, m-health, technology disparities, health, healthy. 
Uma vez realizado o mapeamento terminológico, serão usados os operadores booleanos AND (e), OR (ou) e AND NOT (e não) para construção das estratégias avançadas de busca, onde AND equivale à intersecção, OR equivale à união e AND NOT equivale à exclusão. Assim, para a questão "como engajar na área da saúde de pessoas sem moradia empregando tecnologias da informação e comunicação?” tem-se a seguinte estratégia avançada:

Pesquisa 1: homeless OR unstable housing OR unstably housed OR housing stability OR supportive housing

Pesquisa 2: computer OR computers OR technologies OR communication technology OR technology OR mobile technologies OR ipad OR handheld OR mobile computing OR smart phone OR mobile phone OR iphone OR android OR app OR sms OR text message OR mobile technology OR personal digital assistant OR tablet computer OR informatics OR Internet $\mathrm{OR}$ online OR Mhealth OR m-health OR technology disparities

Pesquisa 3: health OR healthy.

Pesquisa 4: Pesquisa 1 AND Pesquisa 2 AND Pesquisa 3

(homeless OR unstable housing OR unstably housed OR housing stability OR supportive housing) AND (computer OR computers OR technologies OR communication technology OR technology OR mobile technologies OR ipad OR handheld OR mobile computing OR smart phone OR mobile phone OR iphone OR android $\mathrm{OR}$ app OR sms OR text message OR mobile technology OR personal digital assistant OR tablet computer OR informatics OR Internet OR online OR Mhealth OR m-health OR technology disparities) AND (health OR healthy)

Pelos exemplos mencionados, observa-se que o emprego de estratégias de busca e da terminologia possui um impacto decisivo sobre os documentos que serão recuperados nas bases de dados. Recomenda-se, assim, que a estratégia de busca seja registrada e apresentada no relatório final da pesquisa, explicitando-se, dessa forma, informações para que a revisão de literatura seja reproduzível.

\subsection{SELEÇÃO, SISTEMATIZAÇÃO E EQUIPE}

Os documentos encontrados nas bases de dados podem ser incluídos em software específico para a gestão de referências bibliográficas, que facilitará a deduplicação e gestão dos documentos que serão revistos.

Além do software, a seleção dos documentos que comporão a revisão deve ser realizada de forma independente por dois pesquisadores da equipe que aplicarão os critérios que foram estabelecidos no início da revisão. Um terceiro pesquisador será o árbitro caso haja uma seleção 
discrepante. Assim, sugere-se que as revisões sistemáticas sejam elaboradas por, pelo menos, 3 pesquisadores.

O processo de seleção pode ter várias fases. Em um primeiro momento, a seleção pode considerar apenas a leitura dos títulos dos documentos encontrados. Em um segundo momento, pode-se considerar a leitura dos resumos dos documentos encontrados. E em um terceiro momento, pode-se realizar uma análise crítica geral dos documentos encontrados, onde serão observados a coerência do estudo, qualidade metodológica, resultados alcançados, conclusão, financiamento do estudo etc. Todo o processo de seleção deve ser explicitado na forma de fluxo, conforme as diretrizes do PRISMA flow diagram (PRISMA, 2015).

Depois de selecionados os textos, a equipe de revisão precisará lê-los e coletar informações comparáveis como data de realização do estudo, país de realização do estudo, população estudada, intervenção realizada, metodologia empregada, desfechos encontrados. Essas informações podem ser registradas em um quadro para posterior uso no relatório da revisão. Recomenda-se que, desde o início da revisão, se tenha a preocupação de sistematizar as fontes de informação consultadas a fim de que não seja necessário refazer as etapas da revisão e para que os direitos autorais sejam garantidos.

Para pesquisadores que não possuem experiência em revisões de literatura é recomendável incluir na equipe de revisão um ou mais bibliotecários que sejam responsáveis pela gestão das terminologias empregadas no estudo, realização das estratégias de busca e gestão geral dos documentos que serão empregados na revisão, conforme destacado por Spencer e Eldredge (2018). Além do profissional bibliotecário, é recomendável que na equipe da revisão tenha ao menos um especialista na questão que será tratada na revisão. Logo, se a revisão, por exemplo, for sobre religiões africanas, sugere-se que os pesquisadores tenham algum conhecimento prévio no assunto a fim de garantir uma melhor interpretação dos estudos que foram selecionados.

As revisões de literatura sistemáticas são publicadas em forma de artigos científicos ou relatórios científicos ou relatórios executivos com foco em tomadores de decisão no campo da saúde. Além disso, nos últimos anos tem se incentivado que os pesquisadores publiquem uma versão da revisão sistemática em linguagem simples, com foco no público leigo e população em geral. Higgins e Green (2011) explicam no Manual da Cochrane para Revisões Sistemáticas que o resumo em linguagem simples apresenta a revisão em um estilo simples que pode ser entendido pelos consumidores, sendo disponibilizados gratuitamente na Internet. Os resumos de revisões em linguagem simples têm duas partes: um título em linguagem simples e um texto com não mais que 400 palavras. 


\section{REVISÃO SISTEMÁTICA DE LITERATURA: PUBLICAÇÃO}

O modo usual de divulgação de resultados de revisões sistemáticas é a publicação em periódicos científicos. A PubMed, base de dados bibliográfica especializada na área biomédica, registrou no período de um ano entre 01/09/2018 e 31/08/2019 a publicação de 25.624 trabalhos com o termo "systematic review" no título ou abstract (uma média superior a 70 publicações por dia), dos quais 24.795 foram divulgados em artigos de periódicos.

A área da saúde apresenta uma longa tradição na valorização e divulgação de revisões sistemáticas, com organizações dedicadas a este fim. Cochrane é uma dessas organizações internacionais, estabelecida em 1993 como Cochrane Collaboration e com sede em Londres, que tem por lema "Evidências confiáveis. Decisões bem informadas. Melhor saúde." (COCHRANE, 2019a). Para atingir seus objetivos, conta com 11 mil membros e 67 mil apoiadores em 130 países, sendo uma instituição parceira da Organização Mundial da Saúde.

Os resultados das revisões sistemáticas são disseminados tanto em linguagem simples para a população, no sítio Cochrane Evidence (COCHRANE, 2019b), como para profissionais da saúde, na Cochrane Library (COCHRANE, 2019c). Adicionalmente, as revisões são publicadas em um periódico científico, o Cochrane Database of Systematic Reviews (CDSR), que divulga ainda protocolos, editoriais e suplementos, como o Cochrane Methods. Em 2018, o CDSR teve fator de impacto 7,755 no índice InCites (COCHRANE, 2019d).

Outra organização internacional voltada à disseminação de resultados de revisões sistemáticas e outras formas de evidências aplicadas à área da saúde é o Joanna Briggs Institute, estabelecido em 1996 em Adelaide, na Austrália, e que atualmente conta com 70 centros colaboradores em 34 países, incluindo um centro no Brasil (JOANNA, 2019). Assim como o Cochrane, também dissemina suas revisões em uma publicação periódica, o JBI Database of Systematic Reviews and Implementation Reports (LIPPINCOTT, 2019).

No entanto, revisões sistemáticas não são desenvolvidas apenas na área da saúde. A Campbell Collaboration (2019) é uma organização criada em Londres, em 1999, que tem por lema a frase "Melhor evidência para um mundo melhor" e por objetivo promover mudanças sociais e econômicas positivas por meio da elaboração de revisões sistemáticas que possam ser usadas para apoiar as decisões de gestores públicos. São temas contemplados nessas revisões sistemáticas: Educação; Desenvolvimento internacional; Serviço social; Segurança nutricional; Crime e justiça; Negócios e administração; além de tópicos transversais como Métodos de pesquisa e Translação do conhecimento. Assim como as demais organizações voltadas à 
disseminação de revisões sistemáticas, a Campbell Collaboration também divulga seus resultados por meio de uma publicação periódica, a Campbell Systematic Reviews (WILEY, 2019).

É interessante observar que todas essas publicações trazem, em comum, uma demanda pelo rigor metodológico na realização das revisões sistemáticas. Por exemplo, uma revisão sistemática para ser publicada pela Cochrane, deve seguir o preconizado no Cochrane Handbook of Systematic Review of Interventions (HIGGINS; GREEN, 2011), além de ser elaborada por uma equipe com pelo menos dois autores, mas geralmente mais, como forma de reduzir subjetividade e garantir o conhecimento necessário no tema da pesquisa e nos diversos aspectos metodológicos de uma revisão sistemática. Cochrane ainda oferece ferramentas para auxiliar a elaboração da revisão sistemática segundo suas diretrizes, como o Archie, para a gestão de documentos e contatos, e o Review Manager, para facilitar a preparação de revisões, incluindo características e dados dos estudos, bem como tabelas de comparação.

O Instituto Joanna Briggs também provê um conjunto de ferramentas, na forma de checklists, para assegurar a validade de suas revisões sistemáticas e o JBI Database of Systematic Reviews and Implementation Reports, em suas instruções aos autores, afirma que endossa as diretrizes do PRISMA para relatar uma revisão sistemática, além de salientar que manuscritos de revisão sistemática cuja busca tenha sido realizada há mais de um ano são considerados desatualizados e não aceitáveis para publicação.

A Campbell Collaboration, além de utilizar algumas diretrizes e ferramentas oferecidas pela Cochrane, tem também seus checklists para relatos de revisão sistemática e recomendações detalhadas sobre como realizar a etapa da busca (KUGLEY et al., 2017).

Além das revisões sistemáticas endossadas por essas instituições, pesquisas desse tipo são publicadas regularmente nos mais diversos periódicos, como Systematic Reviews, Implementation Science, The Lancet, Journal of the American Medical Association (JAMA) e British Medical Journal (BMJ), na área de saúde, bem como em periódicos interdisciplinares e de outras áreas do conhecimento, tais como PLoS One, International Journal of Information Management, Review of Educational Research, Public Administration, Journal of Data and Information Science e Library Management, entre muitas outras.

Particularmente no campo da biblioteconomia e ciência da informação, Maden e Kotas (2016) fizeram uma revisão das revisões sistemáticas publicadas neste domínio, com foco na avaliação de qualidade reportada pelos próprios autores dos estudos. Para tanto, foram selecionados 40 artigos publicados entre 2003 e 2014 que continham "systematic review" no título, no resumo ou na metodologia. Os estudos selecionados foram em sua maioria publicados 
nos periódicos Health Information and Libraries Journal (14 artigos), Journal of the Medical Library Association (4), Information Research (2), Journal of Academic Librarianship (2), Journal of Documentation (2) e Reference Services Review (2), além de 14 artigos publicados em outros periódicos. Usando os critérios estabelecidos pelo framework PRISMA (2015) e por AMSTAR, atualmente na segunda versão (SHEA et al., 2017), para a apreciação da qualidade dos estudos na revisão sistemática, essas autoras verificaram que a maior parte dos artigos publicados não atenderam aos critérios de revisão sistemática. Assim, concluíram que é preciso ampliar a compreensão sobre os aspectos metodológicos e de qualidade de revisões sistemática nessa comunidade, não apenas entre autores, mas também entre revisores e editores de periódicos da área.

Como qualquer pesquisa realizada com qualidade, um trabalho com uma revisão sistemática rigorosa e atualizada dificilmente será rejeitada por periódicos de qualquer área do conhecimento.

\section{CONCLUSÃO}

No presente estudo, buscou-se apresentar os principais conceitos relacionados à revisão sistemática de literatura, discutindo-se seus componentes como a importância da delimitação da questão de revisão, da seleção adequada da base de dados bibliográficos, do uso adequado das terminologias, da seleção dos documentos que integrarão a pesquisa e aspectos relacionados à composição da equipe de pesquisa. Espera-se que este texto estimule os pesquisadores no desenvolvimento de revisões, já que esta modalidade de pesquisa científica tem ganhado reconhecimento nos diferentes cenários científicos, especialmente, no cenário internacional por seu alto nível de evidência. Igualmente, espera-se que os editores e revisores de manuscritos científicos estejam mais atentos à qualidade das revisões sistemáticas de literatura submetidas à publicação.

Para se aprofundar mais nas questões levantadas, ressalta-se que todas as universidades públicas brasileiras e muitas instituições de ensino superior oferecem a seus alunos disciplinas e cursos relacionados à busca e ao uso da informação para fins de revisão de literatura. Além disso, como o estudo da informação é objeto de muitas ciências (ciência da informação, biblioteconomia, arquivologia e museologia, dedicadas aos fenômenos relacionados à seleção, organização, representação e disseminação da informação), há muita literatura disponível sobre metodologias para prospecção e recuperação da informação para fins científicos. Trata-se assim de uma temática que avança diariamente. 


\section{REFERENCIAS}

BAEK, S. et al. The most downloaded and most cited articles in radiology journals: a comparative bibliometric analysis. European Radiology, v. 28, n. 11, p. 4832-4838, 2018.

CAMPBELL Collaboration. Home page. Londres: Campbell Collaboration, 2019. Disponível em: https://campbellcollaboration.org/. Acesso em: 01 set. 2019.

COCHRANE. About CDSR. Londres: Cochrane, 2019d. Disponível em:

https://www.cochranelibrary.com/cdsr/about-cdsr. Acesso em 01 set. 2019.

COCHRANE. Cochrane Evidence (em português). Londres: Cochrane, 2019b. Disponível em: https://www.cochrane.org/pt/evidence. Acesso em: 01 set. 2019.

COCHRANE. Cochrane Library. Londres: Cochrane, 2019c. Disponível em:

https://www.cochranelibrary.com/. Acesso em: 01 set. 2019.

COCHRANE. Home page. Londres: Cochrane, 2019a. Disponível em:

https://www.cochrane.org/. Acesso em: 01 set. 2019.

COORDENAÇÃO de Aperfeiçoamento de Pessoal de Nível Superior. Portal de periódicos. Brasília: CAPES/MEC, 2018. Disponível em: http://www.periodicos.capes.gov.br. Acesso em: 9 abr. 2018.

CRESWELL, J. W.; CLARK, V. L. P. Designing and conducting mixed methods research. Thousand Oaks, CA: SAGE Publications, 2010.

CRITICAL Appraisal Skills Programme. CASP Systematic Review Checklist. 2018. Disponível em https://casp-uk.net/casp-tools-checklists/. Acesso em: 28 ago. 2019.

GALVAO, M. C. B.; PLUYE, P.; RICARTE, I. L. M. Métodos de pesquisa mistos e revisões de literatura mistas: conceitos, construção e critérios de avaliação. InCID: Revista de Ciência da Informação e Documentação, v. 8, n. 2, p. 4-24, 2017. Disponível em: https://www.revistas.usp.br/incid/article/view/121879. Acesso em: 2 abr. 2018.

GRANT, M. J.; BOOTH, A. A typology of reviews: an analysis of 14 review types and associated methodologies. Health information and libraries journal, v. 26, n. 2, p. 91-108, jun. 2009. Disponível em: http://www.ncbi.nlm.nih.gov/pubmed/19490148. Acesso em: 28 ago. 2019.

HIGGINS, J. P. T.; GREEN, S. (ed). Cochrane Handbook for Systematic Reviews of Interventions. Ed.5.1.0. (s.1.): The Cochrane Collaboration, 2011. Disponível em: www.handbook.cochrane.org. Acesso em 10 abr. 2018.

HONG, Q. N. et al. Proposal: Mixed Methods appraisal tool. Montreal: McGill University, 2018. Disponível em:

http://mixedmethodsappraisaltoolpublic.pbworks.com/w/file/fetch/127916259/MMAT_2018_ criteria-manual_2018-08-01_ENG.pdf. Acesso em: 29 ago. 2019. 
HOWICK, J. et al. Explanation of the 2011 Oxford Centre for Evidence-Based Medicine (OCEBM). Oxford: CEBM, 2011. Disponível em: https://www.cebm.net/2016/05/ocebmlevels-of-evidence/ Acesso em: 2 abr. 2018.

INSTITUTO Nacional de Propriedade Intelectual. Pesquisas. Rio de Janeiro: INPI, 2009. Disponível em: http://www.inpi.gov.br. Acesso em: 9 abr. 2018.

JOANNA Briggs Institute. Home page. Adelaide: JBI, 2019. Disponível em: https://joannabriggs.org/. Acesso em: 01 set. 2019.

KUGLEY, S. et al. Searching for studies: a guide to information retrieval for Campbell systematic reviews. Oslo: The Campbell Collaboration. Disponível em: https://onlinelibrary.wiley.com/doi/pdf/10.4073/cmg.2016.1. Acesso em: 01 set. 2019.

LIPPINCOTT Williams \& Wilkins. JBI Database of Systematic Reviews and Implementation Reports. Alphen aan den Rijn, Holanda: Wolters Kluwer, 2019. Disponível em: https://journals.lww.com/jbisrir/Pages/default.aspx. Acesso em: 01 set. 2019.

MADEN, M.; KOTAS, E. Evaluating approaches to quality assessment in library and information science lis systematic reviews: A methodology review. Evidence Based Library and Information Practice, v. 11, n. 2, p. 149-176, 2016.

POPE C.; MAYS, N.; POPAY, J. Synthesizing quantitative and qualitative health research. Adelaide: Ramsay Books, 2007.

PRISMA Group. Preferred Reporting Items for Systematic Reviews and Meta-Analyses. (s.1.): University of Ottawa/Oxford University, 2015. Disponível em: http://www.prismastatement.org/PRISMAStatement/. Acesso em: 2 abr. 2018.

SHEA, B. J. et al. AMSTAR 2: A critical appraisal tool for systematic reviews that include randomised or non-randomised studies of healthcare interventions, or both. BMJ (Online), v. 358, p. 1-9, 2017.

SIDDAWAY, A. P.; WOOD, A. M.; HEDGES, L. V. How to do a systematic review: a best practice guide for conducting and reporting narrative reviews, meta-analyses, and metasyntheses. Annual Review of Psychology, v. 70, n. 1, p. 747-770, 2019.

SPENCER, A. J.; ELDREDGE, J. D. Roles for librarians in systematic reviews: a scoping. Journal of the Medical Library Association, n.106, v.1, p. 46-56, 2018.

WILEY Online Library. Campbell Systematic Reviews. Hoboken: John Wiley \& Sons. Disponível em: https://onlinelibrary.wiley.com/journal/18911803. Acesso em: 01 set. 2019. 